\title{
0 planejamento estratégico situacional no ensino do gerenciamento em enfermagem
}

\author{
The situational and strategic planning in the teaching of nursing management \\ El planeamiento estratégico situacional en la enseñanza de gerenciamento en \\ enfermeria
}

\author{
Marta Maria Melleiro', Daisy Maria Rizatto Tronchin², Maria Helena Trench Ciampone ${ }^{3}$
}

\begin{abstract}
RESUMO: Trata-se de um estudo documental cujos objetivos foram descrever uma estratégia pedagógica pautada no Planejamento Estratégico Situacional (PES) e mapear os problemas identificados por alunos em um hospital de ensino. Os dados foram obtidos segundo os exercícios de PES realizados no quadriênio 2001-2004. Para a análise dos dados empregou-se a categorização por similaridade do conteúdo, extraindo-se as temáticas: gerenciamento da assistência de enfermagem, recursos humanos, físicos, materiais, normas administrativas, sistema de informação e de referência e contra-referência, apoio logístico, educação em saúde e relacionamento interpessoal. Essa estratégia vem favorecendo o processo ensino-aprendizagem e a integração docente-assistencial, pois emerge do contexto vivenciado pelos alunos e sinaliza aos enfermeiros as áreas que necessitam de investimentos para a resolução de problemas.
\end{abstract}

Descritores: Enfermagem; Ensino; Planejamento estratégico

ABSTRACT: It is a documentary study that aimed to describe the use of the Situational and Strategic Planning as a pedagogical strategy and to identify the problem noticed by the students in a school hospital. The data were collected from the SSP exercises done from 2001 to 2004. They were analyzed by categorizing the context similarity the themes: human, physical and material resources; nursing assistance management; administrative rules and procedures; information system; health education and relationship. This strategy favors the teaching-learning process, because it comes from the student's experiences in the hospital and it tells the nurses the areas that need investment in the solution of the problems.

Keywords: Nursing; Teaching; Strategic planning

RESUMEN: Este estudio documental tuvo como objetivos describir una estrategia pedagógica centralizada en Planeamiento Estratégico Situacional (PES) y mapear los problemas identificados por alumnos en un hospital docente. Los datos fueron obtenidos según los ejercicios de PES realizados en 2001-2004. Para el análisis de los datos fue empleada la categorización por similaridad del contenido, extrayéndose los siguientes temas: gerenciamiento de la asistencia de enfermería, recursos humanos, físicos, materiales, normas administrativas, sistema de información de referencia y de contrareferencia, apoyo logístico, educación en salud y relacionamiento interpersonal. Esta estrategia viene favoreciendo el proceso enseñanza-aprendizaje y la integración docente-asistencial, pues emerge del contexto vivenciado por los alumnos y señala los enfermeros de las áreas que necesitan invertir para la resolución de los problemas.

Descriptores: Enfermería; Enseñanza; Planeamiento estratégico

\section{INTRODUÇÃO}

$\mathrm{O}$ ato de planejar envolve um exercício da razão e da sensibilidade, que engloba atividades de maior ou menor complexidade no cotidiano de trabalho e, sob essa ótica, propicia a construção de planos para enfrentar situações atuais ou futuras.

No processo de trabalho gerencial, o planejamento pode ser definido como um importante instrumento que favorece fazer escolhas e a elaboração de planos que ajudam a enfrentar os processos de mudança. Assim, compreende um conjunto de conhecimentos teóricos e práticos ordenados de modo a possibilitar a interação com a realidade, programar as estratégias e as ações necessárias, para alcançar os objetivos e as metas pre-estabelecidas. Portanto, é um processo contínuo que permite uma postura ativa dos gestores de uma organização na sua relação com os clientes/cidadãos e com o meio ${ }^{(1-2)}$.

\footnotetext{
Professora Doutora do Depto de Orientação Profissional da Escola de Enfermagem da USP. E-mail: melleiro@usp.br

2 Professora Doutora do Depto de Orientação Profissional da Escola de Enfermagem da USP.

3 Professora Associada do Depto de Orientação Profissional da Escola de Enfermagem da USP.
} 
No gerenciamento em enfermagem a função do planejamento configura-se em uma das atividades privativas dos enfermeiros, devido à divisão sócio-técnica do trabalho(3).

Nesse contexto, e acreditando que existe a necessidade de refletirmos sobre como temos executado o planejamento, para que possamos aprimorar tanto o processo como o produto, é que optamos por relatar, neste estudo, a utilização do referencial do Planejamento Estratégico Situacional (PES) no ensino do gerenciamento em enfermagem.

O PES, no campo das políticas públicas, surgiu a partir da rejeição da idéia de uma só racionalidade - a econômica, para a solução das questões políticas e sociais e, também, do reconhecimento da pluralidade de atores sociais em conflito numa realidade complexa e dinâmica. É um instrumento de gestão voltado para a resolução de problemas, no qual os atores sociais participam efetivamente do processo.

O método PES possibilita a explicação de um problema a partir da visão do ator que o declara, a identificação das possíveis causas e a busca por diferentes modos de abordar e propor soluções. Logo, processar problemas implica em explicar como ele nasce e como se desenvolve, fazer planos para atacar as suas causas, analisar e construir a viabilidade política do plano, o que corresponde a ter uma visão real dos problemas locais, sem generalizá-los na descrição e nas propostas de solução.

O problema é algo detectado que incomoda o ator social e o motiva a buscar soluções adequadas, ou seja, aquilo que o ator detecta na realidade e confronta com um padrão que ele considera inadequado ou intolerável e, ainda, o estimula a enfrentá-lo, visando a promoção de mudanças ${ }^{(4)}$.

Para promover mudanças e, conseqüentemente, para que o processo decisório ocorra, é necessário aos diferentes atores envolvidos em uma dada situação, acumular poder, pois sem este são ínfimas ou inexistentes as possibilidades das mudanças serem concretizadas. Alguns autores destacam quatro categorias de poder, que são: o político - representado pelos atores que o detêm em função do exercício de um mandato advindo da posição que ocupam com representatividade social; o econômico - representado pelos atores que detêm os recursos econômicos e financeiros, para intervir no problema podendo ser pessoas físicas ou jurídicas; o administrativo - envolve os atores que possuem a coordenação ou a direção de recursos relacionados à esfera administrativa e o técnico - referente aos atores que abarcam conhecimentos e práticas sobre um determinado tema ${ }^{(4-5)}$.

Esses poderes traduzem-se no controle de recursos escassos, os quais são denominados: conhecimento da informação, capacidade organizativa, capacidade política e capacidade de controle de recursos financeiros ${ }^{(4)}$.
Outro aspecto a ser mencionado, diz respeito aos níveis de controle que os atores sociais detém no processo de resolução da situação-problema, os quais são $a$ governabilidade: onde o controle do ator que declara o problema é total; a influência: na qual o ator tem capacidade de influenciar outros atores que possuem governabilidade sobre os nós explicativos do problema e fora do jogo: onde o ator não tem qualquer controle ou influência sobre as causas do problema.

O PES é desenvolvido por meio de quatro momentos: o explicativo, o normativo, o estratégico e o táticooperacional. A seguir, encontram-se descritos esses momentos, ressaltando-se que a seqüência dos mesmos, deve-se apenas à uma estratégia didática, uma vez que são interdependentes e alternantes.

\section{Momento Explicativo}

No momento explicativo a realidade é descrita mediante a seleção de problemas relevantes, buscando-se a compreensão ampla do porquê esses ocorrem e identificando-se os nós-críticos, que representam os centros práticos de ação.

Em nosso meio, o conhecimento do processo de avaliação de estrutura, processo e resultado, dos recursos epidemiológicos, do sistema de referência e de contra-referência e do fluxo dos usuários atendidos nos serviços de saúde são considerados essenciais para uma explicação dinâmica da situação-problema.

O momento explicativo pode ser sintetizado no que Matus denominou de Fluxograma Situacional ${ }^{(1-2)}$, no qual estão inseridos os seguintes aspectos que resumem o conteúdo dessa etapa:

- A elaboração do problema realizada com clareza e objetividade;

- A presença do ator responsável pela explicação e articulação das ações, no sentido de conceber e operar as intervenções para a solução do problema;

- Os descritores do problema, representados preferencialmente por indicadores de caráter quantitativo, monitoráveis ao longo do tempo e que possibilitem verificar como o problema se comporta numa dada realidade situacional;

- As conseqüências do problema, constando de um exercício de análise e de uma discussão, tendo como produto a síntese dos principais efeitos do problema.

A relevância e a precisão do estabelecimento dos nóscríticos justifica-se pelo fato de que esses serão os focos a serem trabalhados no momento normativo.

\section{Momento Normativo}

Nesta etapa ocorre a identificação dos atores que integram o problema e dos recursos que esses dispõem 
para controlar as operações. Faz-se a projeção de cenários onde são mapeadas as variáveis de teto (as melhores possibilidades), as de centro e as de piso (as piores possibilidades).

A projeção das operações nos três cenários é fundamental, pois considera a relação entre os poderes da instância política, do conhecimento técnico do problema, da capacidade organizativa dos atores e dos recursos financeiros. Portanto, o momento normativo trabalha com a incerteza, sendo necessário, constantemente, avaliar e calcular o futuro, construindo diferentes cenários de atuação ${ }^{(4)}$.

Por conseguinte, é relevante a definição de prazos e metas em relação às operações, tendo-se, dessa forma, a previsão de seu início e de seu término.

\section{Momento estratégico}

Nesta fase, a análise situacional encontra-se focada na construção da viabilidade, mapeando-se todos os atores que possam cooperar ou se opor ao que está sendo proposto, avaliando-se o tipo de controle que cada um detém dos recursos essenciais para o êxito do plano ${ }^{(2)}$.

O momento estratégico busca responder às seguintes indagações: As operações do plano são viáveis nesse momento? Quais as possíveis reações dos atores envolvidos no problema? Como construir a viabilidade para as ações inviáveis?

Cabe ressaltar, que a análise estratégica permeia e deve ser desenvolvida em todos os momentos da elaboração e execução do plano.

\section{Momento tático-operacional}

Consiste no momento de imple-mentação das ações propostas e na adequação às situações apresentadas. Nessa perspectiva, quebra-se a lógica linear - planejar/ executar/avaliar. É preciso recalcular o plano, no qual o monitoramento das operações ajuda a redesenhá-las permanentemente e a avaliação contínua do impacto no processo de organização dos serviços, realimenta a leitura da realidade e da melhor forma de nela intervir ${ }^{(2)}$.

Ao se analisar o PES, podemos descrevê-lo como um método de permanente exercício de diálogo e de reflexão sobre os problemas que incidem em uma dada realidade, visando prever situações e alternativas, antecipar possibilidades de decisão e preparar estratégias para a obtenção de governabilidade sobre as mesmas.

Constata-se que, na análise dos problemas potenciais o PES consiste em um potente instrumento do processo de trabalho gerencial, permitindo ao gestor lançar mão das ações preventivas e contingentes.

Em consonância com essa concepção e acreditando que o planejamento, enquanto instrumento gerencial, deva estar associado à dimensão ético-política no contexto macro ou micro-social, é que os docentes da disciplina de Administração em Enfermagem III, ministrada no $7^{\circ}$ semestre do Curso de Graduação em Enfermagem, da Escola de Enfermagem da USP, vem introduzindo aos alunos o pensamento estratégico, por meio de exposições dialogadas e da operacionalização, em campo de estágio, de um exercício adaptado do PES.

A finalidade dessa estratégia é proporcionar ao aluno o monito-ramento dos problemas identificados e a resolutividade ou não de uma situação vivenciada no campo de estágio e compartilhada com o enfermeiro responsável pela unidade e com os docentes que supervisionam os estágios dessa disciplina.

\section{OBJETIVOS}

- Descrever uma estratégia pedagógica pautada no PES;

- Mapear as situações-problema identificadas pelos alunos no campo de estágio, de um hospital de ensino.

\section{MÉTODO}

Trata-se de um estudo documental, baseado em um levantamento retrospectivo de dados, referentes aos exercícios realizados por alunos no estágio da disciplina de Administração em Enfermagem III. Esse estágio ocorre em um hospital de ensino, do Município de São Paulo, tem aproximadamente 300 horas de duração, distribuídas nos períodos da manhã e da tarde, nas seguintes Divisões: Enfermagem Clínica - DECLI (Seção de Clínica Médica e Unidade de Terapia Intensiva Adulto), Enfermagem Cirúrgica - DEC (Seção de Clínica Cirúrgica e Centro Cirúrgico), Enfermagem Materno-Infantil DEMI (Seção de Centro Obstétrico, Alojamento Conjunto, Berçário, Pediatria e Unidade de Terapia Intensiva Pediátrica e Neonatal) e de Pacientes Externos - DEPE (Seção de Ambulatório, Pronto Socorro Adulto e Infantil), as quais compõem o Departamento de Enfermagem do referido hospital de ensino.

No quadriênio 2001-2004, o estágio de Administração em Enfermagem III foi realizado com 74 grupos, compostos de dois a cinco alunos, sendo 33 grupos na DEMI, 16 na DECLI, 13 na DEC e 12 na DEPE.

Como parte da avaliação da disciplina, cada grupo entrega ao concluir o estágio, um exercício de PES (ANEXO 1) relativo a uma situação-problema detectada no campo de prática, o qual tornou-se o objeto desta análise documental. Para a coleta dos dados, realizada em maio de 2004, foi elaborado um formulário contendo a identificação (unidade, ano e período) e as situações-problema descritas nesses exercícios.

A operacionalização da coleta de dados deu-se em dois momentos: no primeiro foi solicitado aos docentes 
que acompanharam os alunos, uma cópia dos exercícios correspondentes ao referido quadriênio, os quais foram agrupados de acordo com as unidades de origem. No segundo momento, os problemas foram compilados, realizando-se a categorização de acordo com a similaridade do conteúdo, sendo extraídas as temáticas a seguir: gerenciamento da assistência de enfermagem, recursos humanos, físicos, materiais, normas administrativas, sistema de informação e de referência e de contra-referência, apoio logístico, educação em saúde e relacionamento interpessoal.

\section{RESULTADOS E DISCUSSÕES}

Do total de 74 exercícios previstos, foram obtidos $65(88,0 \%)$, provenientes das seguintes divisões: 31 $(47,7 \%)$ da DEMI, $14(21,5 \%)$ da DECLI, $11(16,9 \%)$ da DEPE e $9(13,9 \%)$ da DEC.

A Figura 1 demonstra os exercícios previstos e os obtidos correspondentes aos grupos de alunos que freqüentaram o campo de estágio em Administração em Enfermagem III no período de 2001 a 2004.

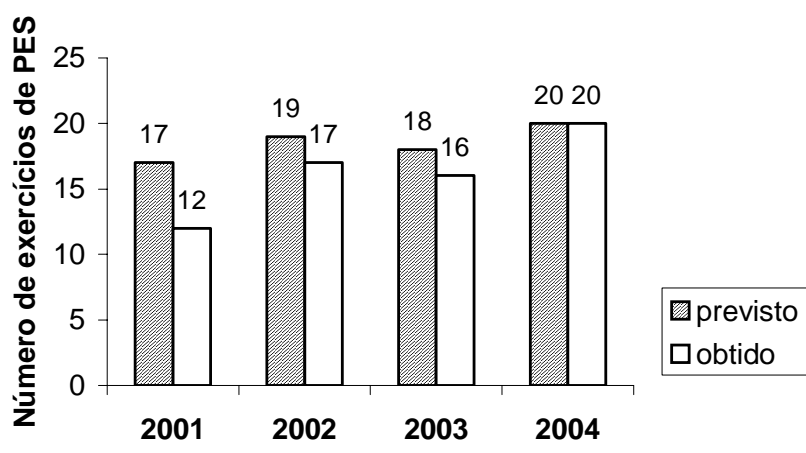

Figura 1 - Distribuiçäo dos exercicios de PヒS realizados no quadriênio 2001-2004. São Paulo. 2004.

Observa-se na Figura 1 que em 2001 houve uma maior defasagem na relação exercícios previstos-obtidos, totalizando $70,5 \%$ dos dados; em contrapartida em 2004 foram obtidos $100 \%$ dos exercícios.

Na Tabela 1, estão relacionados os problemas identificados nos campos de estágio, de acordo com a temática gerencial.

Tabela 1 - Distribuição dos problemas identificados no exercício PES, no quadriênio 2001-2004, segundo a temática gerencial. São Paulo, 2004.

\begin{tabular}{lrr}
\hline TEMÁTICA GERENCIAL & N & $\%$ \\
\hline Gerenciamento da assistência de enfermagem: & 12 & 18,5 \\
Procedimentos de enfermagem & 8 & 12,3 \\
Operacionalização do SAE & 9 & 13,8 \\
Gerenciamento de recursos humanos & 9 & 13,8 \\
Gerenciamento de recursos materiais & 7 & 10,9 \\
Sistema de informação & 6 & 9,3 \\
Normas administrativas & 5 & 7,7 \\
Apoio logístico & 3 & 4,6 \\
Relacionamento interpessoal & 3 & 4,6 \\
Programas e ações de saúde & 2 & 3,0 \\
Gerenciamento de recursos físicos & 1 & 1,5 \\
Sistemas de referência e de contra-referência & 65 \\
\hline Total & 2 \\
\hline
\end{tabular}

Pelos dados da Tabela 1, constata-se que dentre os problemas detectados, a maioria $20(30,8 \%)$ está relacionada ao gerenciamento da assistência de enfermagem, sendo que $12(18,5 \%)$ e $8(12,3 \%)$ envolvem procedimentos de enfermagem e a operacionalização do Sistema de Assistência de Enfermagem (SAE). A seguir, encontram-se os referentes aos recursos humanos e aos materiais, sobretudo aspectos relativos ao dimensionamento de pessoal e a escassez de materiais de consumo e 7(10,9\%) concernentes ao sistema de informação.

Tendo em vista, que o gerenciamento da assistência de enfermagem pressupõe o direcionamento das atividades da equipe de enfermagem para a organização do processo cuidativo e, conseqüentemente, para o alcance da qualidade assistencial, torna-se imperativo que os dirigentes dos serviços de saúde, gerem novas e criativas estratégias, visando capacitar a equipe de enfermagem no desenvolvimento de atividades técnicas e intelectuais. Essa capacitação se faz necessária, em virtude do SAE ser um processo dinâmico, que demanda raciocínio clínico para analisar, avaliar e tomar decisões acerca das condutas.

Acredita-se que os docentes e enfermeiros de campo devam ter o compromisso de planejar conjuntamente as atividades do processo ensino-aprendizagem, para que a complementaridade do mesmo, conduza a resultados efetivos e eficazes.

Quanto aos recursos humanos e materiais, os dados obtidos sinalizam que a dimensão da estrutura encontra-se vulnerável, devendo, portanto, ser foco de 
atenção, uma vez que a estrutura é um componente de avaliação dos serviços e mantém estreita relação com a qualidade assistencial $^{(6)}$.

Assim, sob a ótica gerencial, é preciso identificar alternativas para estruturar os problemas e pensar opções para sua solução em diferentes ce- nários, em que há diversos atores sociais e jogos de força em ações que podem ser utilizados em inúmeras circunstâncias ${ }^{(7)}$.

A Tabela 2 demonstra os problemas detectados nos exercícios de PES por ano.

Tabela 2 - Distribuição dos problemas dos exercícios de PES, no quadriênio 2001-2004, segundo a temática gerencial e o ano de ocorrência. São Paulo, 2004.

\begin{tabular}{|c|c|c|c|c|}
\hline & 2001 & 2002 & 2003 & 2004 \\
\hline Temática gerencial & $\mathrm{n}=12$ & $\mathrm{n}=17$ & $\mathrm{n}=16$ & $\mathrm{n}=\mathbf{2 0}$ \\
\hline \multicolumn{5}{|l|}{ Gerenciamento da assistência de enfermagem: } \\
\hline Procedimento de enfermagem & 2 & 6 & 3 & 1 \\
\hline Operacionalização do SAE & 2 & 1 & 1 & 4 \\
\hline Gerenciamento de recursos materiais & 2 & 3 & 1 & 3 \\
\hline Gerenciamento de recursos humanos & 2 & 3 & 4 & - \\
\hline Normas administrativas & 2 & 2 & 2 & - \\
\hline Gerenciamento de recursos físicos & - & - & - & 2 \\
\hline Relacionamento interpessoal & - & 1 & 2 & - \\
\hline Programas e ações de saúde & - & - & 2 & 1 \\
\hline Apoio logístico & 2 & - & - & 3 \\
\hline Sistema de referência e de contra-referência & - & - & 1 & - \\
\hline Sistema de informação & - & 1 & - & 6 \\
\hline
\end{tabular}

Verifica-se que em 2001 e em 2003 os problemas detectados encontram-se distribuídos uniformemente nas diversas temáticas, destacando-se em 2002 os problemas referentes ao gerenciamento da assistência de enfermagem, principalmente, os de procedimentos de enfermagem e em 2004 os de sistema de informação.

O sistema de informação é um conjunto de procedimentos organizados que, quando executados, provêem informação de suporte à organização ${ }^{(8)}$. Dessa maneira, constituem-se como características fundamentais desse sistema: a sua sistematização e a sua disponibilidade. Isto posto, as informações são apresentadas aos usuários da organização, os clientes externos e os internos, os quais são os responsáveis por sua interpretação.
As informações em saúde constituem ferramenta essencial para definir estratégias para a consecução de melhorias nas condições dos serviços de saúde. Assim, quando há falhas no gerenciamento desse sistema e quando o gestor não está familiarizado com essa temática, certamente haverá comprometimento dos dados gerados e de sua qualidade ${ }^{(9)}$.

A Tabela 3 apresenta os dados da relação exercíciolocal de ocorrência, observando-se que a DEMI apresenta um maior quantitativo de problemas, uma vez que devido ao número de seções que contempla, recebeu um maior número de estagiários, que desenvolveram um maior número de exercícios.

Tabela 3 - Distribuição dos problemas dos exercícios de PES, em 2001-2004, segundo a temática gerencial e o local de ocorrência. São Paulo, 2004.

\begin{tabular}{|c|c|c|c|c|}
\hline Temática gerencial & $\begin{array}{l}\text { DECLI } \\
n=14\end{array}$ & $\begin{array}{l}\text { DEMI } \\
\mathrm{n}=31\end{array}$ & $\begin{array}{l}\text { DEC } \\
n=8\end{array}$ & $\begin{array}{l}\text { DEPE } \\
\mathrm{n}=12\end{array}$ \\
\hline \multicolumn{5}{|l|}{ Gerenciamento da assistência de enfermagem: } \\
\hline Operacionalização do SAE & 4 & 2 & 1 & 1 \\
\hline Procedimento de enfermagem & 5 & 5 & - & 2 \\
\hline Sistema de informação & 1 & 1 & 4 & 1 \\
\hline Normas administrativas & 1 & 2 & - & 3 \\
\hline Gerenciamento de recursos materiais & - & 5 & 3 & 1 \\
\hline Gerenciamento de recursos humanos & 2 & 6 & - & 1 \\
\hline Apoio logístico & - & 4 & - & 1 \\
\hline Programas e ações de saúde & - & 3 & - & - \\
\hline Relacionamento interpessoal & 1 & 2 & - & - \\
\hline Gerenciamento de recursos físicos & - & 1 & - & 1 \\
\hline Sistema de referência e de contra-referência & - & - & - & 1 \\
\hline
\end{tabular}


Constata-se por meio da Tabela 3 que na DECLI e na DEMI os problemas referentes ao gerenciamento da assistência de enfermagem são os mais incidentes; enquanto que na DEC sobressaem-se os relativos à sistema de informação e na DEPE observa-se quantitativos similares nas temáticas gerenciamento da assistência de enfermagem e normas administrativas. Ao se relacionar os dados desta tabela com a Tabela 2 pode-se inferir que o maior quantitativo de problemas detectados reporta-se ao gerenciamento da assistência de enfermagem, ratificando a necessidade de propiciar condições de capacitação e desenvolvimento dos recursos humanos.

\section{CONSIDERAÇÕES FINAIS}

Ao longo desses anos, constata-se que o PES vem se constituindo em um recurso pedagógico importante, levando o aluno a aplicar o pensamento crítico e reflexivo para explicar a realidade, descrever a maneira de conceber e tornar factíveis os planos propostos, em distintos cenários.

Cabe salientar, que na elaboração do exercício, ocorre a parceria entre os enfermeiros e alunos na declaração do problema e, dessa forma, a apresentação do exercício no campo de prática, tem possibilitado a implementação de ações viáveis, com base no contexto vivido. Assim, destaca-se a relevância dessa estratégia por romper com uma visão idealizada da realidade onde as contingências costumam ser pouco exploradas, fazendo com que em muitas situações os trabalhos desenvolvidos por alunos fiquem apenas como exercícios didáticos.

Frente ao exposto, é vital que por meio da integração docente-assistencial sejam realizados fóruns de discussões para a troca de informações e experiências, onde docentes, alunos e profissionais que atuam e participam, direta ou indiretamente, do gerenciamento desses serviços, estejam conscientes de sua responsabilidade e de sua governabilidade acerca dos problemas detectados e de seu papel para o alcance dos objetivos e das metas institucionais.

\section{REFERÊNCIAS}

1. Tancredi FB, Barrios SRL, Ferreira JHGF. Planejamento em saúde. São Paulo: Faculdade de Saúde Pública; 1998. [Série Saúde e Cidadania].

2. Almeida ES, Vieira CL, Castro CGJ, Furtado LC, Inojosa RM. Planejamento e programação em saúde. In: Westphal M.F, Almeida ES. (organizadores.) Gestão de serviços de saúde. São Paulo: EDUSP; 2001. p. 255-72.

3. Ciampone MHT, Melleiro MM, Silva MRB, Pereira I. Processo de planejamento na prática da enfermagem em um hospital de ensino. Rev Esc Enferm USP 1998; 32(3):273-80.

4. Matus C. Política, planejamento \& governo. Brasília: IPEA;1996.

5. Testa M. Pensamento estratégico e a lógica da programação: o caso da saúde. São Paulo: Hucitec; 1995.

6. Donabedian A. The role of outcomes in quality assessment and assurance. Qual Rev Bull 1992; 20(6):975-92.

7. Malik AM, Schiesari LMC. Gestão de recursos humanos. São Paulo: Faculdade de Saúde Pública; 1998. [Série Saúde e Cidadania].

8. Carvalho A, Eduardo MBP. Sistemas de informação em saúde para municípios. São Paulo: Faculdade de Saúde Pública; 1998. [Série Saúde e Cidadania].

9. Soboll MLMS, Carvalho A, Eduardo MBPE, Tanaka O. Sistemas de informação em saúde. In: Westphal MF, Almeida ES, organizadores. Gestão de serviços de saúde. São Paulo: EDUSP; 2001. p. 205-254. 


\section{ANEXO 1}

FORMULÁRIO DO EXERCíCIO DE PLANEJAMENTO ESTRATÉGICO SITUACIONAL (PES)

\section{ETAPA 1}

- Descreva uma situação problemática percebida durante o estágio para ser analisada segundo o referencial do Planejamento Estratégico Situacional.

- Liste as possíveis causas do problema utilizando o formulário.

Instituição:

Unidade:

Problema detectado:

Atores que declaram:

\section{POSSÍVEIS CAUSAS DO PROBLEMA}

\section{EFEITOS/CONSEQUÊNCIAS DO PROBLEMA}

\section{DESCRITORES DO PROBLEMA}

\section{ETAPA 2}

- Correlacione as possíveis causas do problema com os atores e o nível de governabilidade deles sobre o problema.

- Descreva as conseqüências/efeitos e os descritores do problema.

\begin{tabular}{|c|c|c|}
\hline POSSIVEIS CAUSAS & ATORES & NÍVEIS DE CONTROLE* \\
\hline & & \\
\hline
\end{tabular}

${ }^{\star} \mathrm{G}=$ Governabilidade; I = Influência e FJ = Fora do Jogo.

\section{ETAPA 3}

- Liste os nós críticos (centros práticos de ação) correlacionando-os com as operações e demandas de ações e os respectivos atores responsáveis e os recursos que controlam.

\begin{tabular}{|l|l|c|c|c|}
\hline Nó-Crítico & Nós-críticos/Descrição & $\begin{array}{c}\text { Operações/Demandas } \\
\text { de Ações }\end{array}$ & Ator Responsável & Recursos que Controla** \\
\hline
\end{tabular}

** Conhecimento do problema/informação; capacidade organizativa; capacidade política e capacidade de controle de recursos financeiro

\section{ETAPA 4}

- Visualize e descreva os possíveis cenários e as respectivas operações viáveis.

\begin{tabular}{|l|l|}
\hline CENÁRIO DE TETO (MELHORES): & OPERAÇÕES VIÁVEIS (TOTAL/PARCELAMENTO) \\
\hline CENÁRIO DE CENTRO: & OPERAÇÕES VIÁVEIS: \\
\hline CENÁRIO DE PISO (PIORES): & OPERAÇÕES VIÁVEIS: \\
\hline
\end{tabular}

